\title{
C Research Square \\ Expression of Efflux Pumps, Porins and Genotypic Insight Into the Carbapenem Resistance in Acinetobacter Baumannii
}

\section{Alka Hasani}

Tabriz University of Medical Sciences

Abolfazl vahhabi ( $\square$ vahhabi.a@gmail.com )

Tabriz University of Medical Sciences Faculty of Medicine https://orcid.org/0000-0001-5123-8156

\section{Mohammad Ahangarzadeh Rezaee}

Tabriz University of Medical Sciences

\section{Behzad Baradaran}

Tabriz University of Medical Sciences

\section{Akbar Hasani}

Tabriz University of Medical Sciences

\section{Hossein Samadi Kafil}

Tabriz University of Medical Sciences

\section{Faeze Abbaszadeh}

Tabriz University of Medical Sciences

\section{Research}

Keywords: Acinetobacter baumannii, Carbapenem, Carbapenemase, Oxacillinase, $\beta$-lactamase, Multi Drug Resistance, Multiplex Polymerase Chain Reaction

Posted Date: June 25th, 2021

DOI: https://doi.org/10.21203/rs.3.rs-573246/v1

License: (c) (1) This work is licensed under a Creative Commons Attribution 4.0 International License. Read Full License 


\section{Abstract \\ Background.}

The emergence of multidrug and extensive drug resistant strains of Acinetobacter baumannii is a multifactorial consequence. Carbapenems, once considered the traditional standard of care for Acinetobacter infections however, are gradually being curtailed from the therapeutic regime due to the emergence of Carbapenem resistant Acinetobacter baumannii (CRAB). Several carbapenem resistant mechanisms have been postulated for the rise of CRAB. This study investigated clinical A.baumannii isolates for the presence and level of expression of enzymatic and non-enzymatic genes, putatively associated with carbapenem resistance and their association with sequence typing.

\section{Methods.}

Uniplex, and Multiplex PCR were performed to identify the presence of oxacillinase (OXA) and metallo $\beta$ lactamase (MBLs) genes respectively. The level of expression of efflux pumps (adeB and adeJ) and porins (carO, omp33-36 and oprD) was investigated by Real-time PCR.

\section{Results.}

Of the 112 isolates obtained during this study, $100 \%$ were multidrug-resistant and $48.2 \%$ were extensive drug-resistant A.baumannii. All CRAB isolates harbored b/aXA-51-like, while, $82.1 \%$ and $63.4 \%$ of these isolates carried b/aXX-23-like and bla OXA-24/40-like genes, respectively. In contrast, the frequency of metallo $\beta$-lactamase genes was comparatively less than the oxacillinase genes. Over-expression of adeB and ade $J$ was observed in $66 \%$ and $42.8 \%$ A.baumannii strains respectively, while, decreased expression of carO, omp33-36 and oprD was observed in $75 \%, 66 \%$ and $72.3 \%$ strains respectively.

\section{Conclusion.}

Consistent with that reported by others, our study highlights the significant dissemination of the oxacillinase, bla $a_{0 X A-23-l i k e}$ in CRAB isolates, particularly the simultaneous occurrence of bla OXA-23-like with bla $a_{\mathrm{OXA}-40}$. Interestingly, while changes in the expression of efflux pumps and porins were observed nevertheless, more in depth investigation is required to decipher their contribution to carbapenem resistance in these strains.

\section{Introduction}

Acinetobacter baumannii is an important opportunistic pathogen causing nosocomial infections, especially in patients admitted to intensive care (ICU) and burn units $(1,2)$. Clinical A.baumannii isolates 
are routinely resistant to several antimicrobial classes, including cephalosporins, fluoroquinolones and carbapenems, rendering infections difficult to eradicate $(2,3)$. The wide antibiotic resistance may be because of the organism's propensity to acquire resistance determinants carried by plasmids, transposons and/or resistance islands $(1,2)$. Carbapenems, mainly imipenem and meropenem, were until recently the drug of choice for the treatment of A.baumannii infections, due to their broad spectrum antibacterial activity and minimal side effects. However, their overuse and/or misuse has led to an increasing prevalence of carbapenem resistant A.baumannii (CRAB) posing a pragmatic problem worldwide (1-3).

The mechanisms underlying resistance to carbapenems include, hydrolysis by $\beta$-lactamase enzymes, overexpression of efflux pumps, alterations in porins and changes in penicillin-binding proteins $(4,5)$. Enzymatic degradation by carbapenem-hydrolyzing class $\mathrm{D}$ $\beta$-lactamases (CHDLs, Ambler class $\mathrm{D}$ ) encoded by oxacillinase (OXA) producing genes comprising bla $a_{\text {OXA-51-like, }}$ bla OXA-23-like, $b / a_{\text {OXA-24/40-like, }}$ bla $a_{\text {OXA-58-like, }}$ bla OXA-143-like, $_{\text {b }} a_{\text {OXA-235-like }}$ and metallo $\beta$-lactamases (MBLs, Ambler class B) encoded by $b / a_{\mathrm{IMP}}, b / a_{\mathrm{SIM}}, b / a_{\mathrm{VIM}}, b / a_{\mathrm{GIM}}, b / a_{\mathrm{SPM}}, b / a_{\mathrm{NDM}}$ are considered the most prevalent mechanism of carbapenem resistance in A.baumannii, nevertheless, this etiology may vary geographically (4-6).

Overexpression of efflux pump genes is recognized as a key mechanism for the reduction of intracellular antibiotic concentrations, promoting drug resistance in many bacterial genera $(7,8)$. The Resistance Nodulation Cell Division (RND) systems are the most clinically relevant efflux pumps in Gram-negative bacteria including, A.baumannii. Expression of them controls the biofilm formation as well as antibiotic resistance mechanism $(8,9)$. To date, overexpression of three RND systems, including AdeABC, AdelJK and AdeFGH has been associated with multidrug resistance (MDR) in A.baumannii. The AdeABC pump was the first characterized RND type superfamily which consists of AdeA (a periplasmic membrane fusion protein), AdeB (an inner membrane RND transporter) and AdeC (an outer membrane porin) (7-9). Synergy between $\beta$-lactamases and efflux pumps has been implicated in A.baumannii strains with higher levels of resistance to $\beta$-lactams, including carbapenems $(10,11)$.

Variation in the structure and/or expression of porins is another mechanism indicted for promoting carbapenem resistance in A.baumannii. Reduced expression of the carbapenem-associated outer membrane proteins, evidenced as CarO, Omp33-36 kDa and OprD homologue is accountable for CRAB (8, 12). The CarO is the most characterized porin in A.baumannii, and alteration in its expression reduces the penetration of imipenem into the cell, promoting the drug resistance $(5,13)$. Meropenem resistance, however, may be mediated by another porin-mediated pathway (Omp33-36 kDa), given the absence of a meropenem binding site on CarO $(8,14,15)$. The OprD homologue porin is an attractive candidate for a carbapenem-specific channel and it displays $49 \%$ amino acid similarity to Pseudomonas aeruginosa OprD protein associated with carbapenem resistance, whereby its loss can induce imipenem and meropenem susceptibility $(16,17)$. However, few published studies are available on the functions of the OprD homologue and its involvement in carbapenem resistance in A.baumannii $(15,18,19)$. 
In this study, we aimed to assess the probable mechanisms implicated in the carbapenem resistance in A.baumannii clinical isolates recovered from University teaching hospital of Tabriz University of Medical Sciences, Northwest of Iran, including the presence of carbapenemase enzymes (OXAs and MBLs) and expression of RND efflux pumps components (AdeB and AdeJ) and porins (CarO, Omp33-36 and OprD).

\section{Materials And Methods}

\section{Bacterial isolates}

One hundred and twelve A. baumannii isolates collected from 57.1\% $(n=65)$ male and $42.9 \%(n=47)$ females in-patients admitted to different hospital wards between October 2018 to October 2019 were included in this study. If A.baumannii was isolated from other clinical specimen of the same patient, only one isolate per patient was included in the study. Wound specimens revealing A.baumannii as few colonies or urine specimen reporting presence of this organism in count less than $10^{5} \mathrm{CFU} / \mathrm{ml}$ with normal white blood cell count were excluded from the study. Ethical Committee of Tabriz University of Medical Sciences approved the project under the code: IR.TBZ MED.VCR.REC.1397.042.

\section{Identification and antimicrobial susceptibility testing}

A.baumannii isolates were identified using classical biochemical methods including, growth characterization on blood and MacConkey agar, oxidase test, glucose oxidation, citrate utilization and growth at $37^{\circ} \mathrm{C}$ and $44^{\circ} \mathrm{C}(20)$. PCR amplification of gyrB and $r p o B$ genes (Supplementary Table 1) subsequently confirmed $A$.baumannii at genotypic level $(21,22)$. The disk diffusion method was used to evaluate susceptibility to the following antimicrobial agents: imipenem $(10 \mu \mathrm{g})$, meropenem $(10 \mu \mathrm{g})$, doripenem $(10 \mu \mathrm{g})$, ceftazidime $(30 \mu \mathrm{g})$, cefotaxime $(30 \mu \mathrm{g})$, ceftriaxone $(30 \mu \mathrm{g})$, ciprofloxacin $(5 \mu \mathrm{g})$, levofloxacin $(5 \mu \mathrm{g})$, gentamicin $(10 \mu \mathrm{g})$, amikacin $(30 \mu \mathrm{g})$, tobramycin $(30 \mu \mathrm{g})$, trimethoprimsulfamethoxazole $(1.25 / 23.75 \mu \mathrm{g})$, piperacillin/tazobactam $(100 / 10 \mu \mathrm{g})$, and ampicillin/sulbactam $(10 / 10 \mu g)(L i o f i l c h e m$, Italy). The results were interpreted according to Clinical and Laboratory Standards Institute (CLSI, 2018) guidelines (23). A.baumannii isolates were defined as Multi drug resistant (MDR) and Extremely drug resistant (XDR) according to the International Expert Proposal for Interim Standards Guidelines (24).

Minimal inhibitory concentration (MICs) were determined by E-test for imipenem, meropenem, and doripenem as well as ampicillin-sulbactam (Liofilchem, Italy) and broth dilution using Mueller-Hinton broth for colistin according to the manufacturer's instructions and the (CLSI, 2018) guidelines, respectively (23). Escherichia coli ATCC 25922 and Pseudomonas aeruginosa ATCC 27853 obtained from Iranian Biological resource center, Tehran, were used as control strains.

\section{PCR and quantitative Real time PCR experiments}

Quantitative real time PCR was conducted to evaluate the expression levels of the efflux pumps (adeB and adeJ) and porins (carO, omp33-36 and oprD) using specific primers described previously (25, 
26) (Supplementary Table 1). Briefly, A.baumannii cultures were grown to exponential phase on Mueller Hinton agar plate in aerobic condition, at $37^{\circ} \mathrm{C}$ for $18-24 \mathrm{hrs}$ and then total RNA was extracted using QIAGEN RNeasy kit (QIAGEN, Germany). DNase-treated RNA (>50 $\mathrm{ng} / \mu \mathrm{l}$ ) from each bacterial isolate was converted to cDNA using 2-steps reverse transcriptase kit (containing MMLV reverse transcriptase enzyme) (Takara Bio, Japan) following the manufacturer's protocol. RT-PCR was performed using SyberGreen mastermix (Amplicon, Denmark) in Rotorgen real time PCR (Rotorgene-Corbett-6000, Australia), with the following cycling conditions: $3 \mathrm{~min}$ at $94^{\circ} \mathrm{C}$, followed by 35 cycles of $30 \mathrm{sec}$ at $94^{\circ} \mathrm{C}$, $45 \mathrm{sec}$ at $56^{\circ} \mathrm{C}$, and $60 \mathrm{sec}$ at $72^{\circ} \mathrm{C}$ pursued by $5 \mathrm{~min}$ at $72^{\circ} \mathrm{C}(25,26)$. The results were normalize to the expression of the housekeeping gene, $r p o B(22)$. A.baumannii strain ATCC 19606 obtained from Iranian Biological resource center, Tehran, was used as the reference and expression analysis calculated as relative expression of the mRNA.

Uniplex or multiplex PCR was performed to detect OXA genes (b/aXA-51-like, bla OXA-23-like, $b / a_{\text {OXA-24/40-like, }}$ $b / a_{\text {OXA-58-like, }}, b / a_{\text {OXA-143-like }}$ and bla $\left.a_{\text {OXA-235-like }}\right)$, MBLs genes (b/a $a_{I M P}, b / a_{\mathrm{VIM}}, b / a_{\mathrm{SIM}}, b / a_{\mathrm{GIM}}, b / a_{\mathrm{SPM}}$ and $\left.b / a_{\mathrm{NDM}}\right)$ and the insertion sequence IS $A b a 1$, using specific primers as previously described (2732) (Supplementary Table 1). PCR mapping experiments using IS $A b a 1$ forward $/$ bla $/ a_{\text {XXA-51-like }}$ or $b / a_{\text {OXA-23- }}$ like reverse primers (ISAba $1 \mathrm{~F} /$ bla $a_{\text {OXA-51-like }}$ or bla $a_{\text {OXA-23-like }} \mathrm{R} P C R$ ) were performed using $2 X-P C R$ Master mix (Amplicon, Denmark) and the bacterial genomic DNA under following cycling conditions: 3 min at $94^{\circ} \mathrm{C}$, followed by $35 \mathrm{cycles}$ of $30 \mathrm{sec}$ at $94^{\circ} \mathrm{C}, 45 \mathrm{sec}$ at $56^{\circ} \mathrm{C}$, and $60 \mathrm{sec}$ at $72^{\circ} \mathrm{C}$ pursued by $5 \mathrm{~min}$ at $72^{\circ} \mathrm{C}$ using thermocycler (Bio-Rad, UK) $(27,31)$. PCR products were analyzed by electrophoresis on $1.5 \%$ agarose gel (Merck, Germany) in 1X Tris-Borate-EDTA (TBE) buffer at 90 volts for 60 min $(27,31)$.

To determine the clonal lineage multiplex PCRs were designed to selectively amplify SG1 (EU clone II) and SG2 (EU clone I) alleles encoding ompA, csuE, and bla OXA-51-like genes as described previously (33).

\section{Statistical analysis}

Categorical variables were compared by the $\chi^{2}$ or Fisher's exact test and differences in means were assessed by Student's t-test using SPSS 22.0 statistical software (SPSS Inc. Chicago, IL). A statistically significant difference was considered as a $P$-value $<0.05$.

\section{Results}

\section{Source of bacterial isolates and identification as A.baumannii}

Bacterial isolates identified as A.baumannii on the basis of biochemical tests were subjected to PCR assay for the amplification of $g y r B$ and $r p o B$ genes to confirm the clinical isolates genotypically. All isolates revealed the presence of 294 and $490 \mathrm{bp}$ and $350 \mathrm{bp}$ bands compatible to the presence of $g y r B$ and $r p o B$ genes respectively and confirming them as $A$.baumannii. The source of these isolates was: wound (33.9\%), endotracheal aspirate (28.6\%), blood (19.6\%), urine (10.7\%), broncho-alveolar lavage 
$(4.5 \%)$ and IV catheter (2.7\%) obtained from patients admitted in various wards: urology $(n=5)$, burn $(n=5)$, infectious diseases $(n=5)$ and different ICU wards ( $n=97)$ (Supplementary Table 2).

\section{Disk agar diffusion assay and phenotypic detection of antibiotic resistant profile}

When results of disk agar diffusion were analyzed, all A.baumannii isolates could be grouped in four antimicrobial resistant profiles (Figure 1). Noteworthy, all A.baumannii isolates were found resistant to ceftazidime, cefotaxime, ceftriaxone, imipenem, meropenem, doripenem, ciprofloxacin, levofloxacin, trimethoprim-sulfamethoxazole, and piperacillin/tazobactam. Thus, all isolates were considered MDR and CRAB strains. In addition, resistance to gentamicin, and amikacin, tobramycin and ampicillin/sulbactam was observed in 72.3\% ( $n=81), 64.3 \%(n=72), 48.2 \%(n=54)$ isolates, respectively. Among them, $51.8 \%(n=58)$ isolates showed resistant to all classes of antibiotics except colistin and ampicillin/sulbactam, and thus, were designated as XDR strains.

\section{Antimicrobial susceptibility testing by MIC assay}

To determine the lowest concentration of carbapenems and ampicillin-sulbactam that prevents visible growth of a microorganism, MIC assay was performed using E-test strips. All A.baumannii isolates were confirmed resistant to imipenem, meropenem, and doripenem ( $\mathrm{MIC}>32 \mu \mathrm{g} / \mathrm{mL})$ thus defining them as CRAB while all isolates retained their susceptibility towards ampicillin- sulbactam ( $\mathrm{MIC}<2 \mu \mathrm{g} / \mathrm{mL})$. When A.baumannii isolates were checked for their susceptibility towards colistin by broth dilution method, all isolates were observed susceptible $(\mathrm{MIC}<2 \mu \mathrm{g} / \mathrm{mL})$.

\section{Screening of CRAB strains for carbapenemase genes and insertion sequence ISAba1}

All CRAB strains were screened for the presence of OXA genes (bla OXA-51-like, $b / a_{\text {OXA-23-like, }}, b / a_{0 X A-24 / 40-l i k e,}$ $b / a_{\text {OXA-58-like, }}, b / a_{\text {OXA-143-like }}$ and $\left.b / a_{\text {OXA-235-like }}\right)$, MBLs genes (b/a $a_{\mathrm{IMP}}, b / a_{\mathrm{VIM}}, b / a_{\mathrm{SIM}}, b / a_{\mathrm{GIM}}, b / a_{\mathrm{SPM}}$ and $\left.b / a_{N D M}\right)$ and the insertion sequence ISAba1 so as to assess the origin of carbapenem resistance in the above mentioned strains by performing either uniplex or multiplex PCRs depending upon the PCR amplification conditions.

All CRAB strains were positive for the b/a $a_{\text {XXA-51-like }}$ gene. Presence of $b / a_{\text {OXA-23-like }}$ and $b / a_{0 X A-24 / 40-l i k e}$ genes was observed in $82.1 \%(n=92)$ and $36.6 \%(n=41)$ CRAB strains respectively. No CRAB strain was positive for bla $a_{\text {OXA-58-like, }}$ bla $a_{\text {OXA-143-like }}$ or bla OXA-235-like. Co-existence of bla $a_{\text {OXA-23-like, }} b / a_{\text {OXA-51-like }}$ and

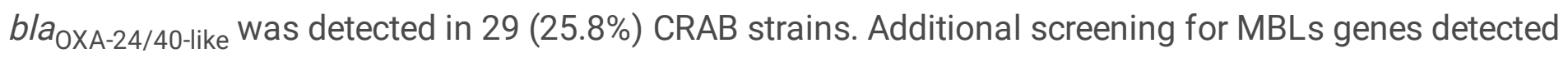
presence of $b / a_{\mathrm{NDM}}(6.2 \% ; \mathrm{n}=7)$ and $b / a_{\mathrm{IMP}}(4.5 \% ; \mathrm{n}=5)$ while, no CRAB strain was observed positive for $b / a_{\mathrm{VIM}}, b / a_{\mathrm{SIM}}, b / a_{\mathrm{GIM}}$ and $b / a_{\mathrm{SPM}}$. The ISAba1 element was present in the majority $(95.5 \% ; \mathrm{n}=107)$ of CRAB strains and found to be upstream of bla $a_{\text {OXA-23-like }}$ in 64 (69.5\%) bla $a_{\text {OXA-23-like }}$-producing CRAB strains. However, the ISAba 1 element was not found upstream of bla OXA-51-like positive CRAB strains.

\section{Quantitative real time experiment for adeB and ade $J$ efflux pump genes expression}


CRAB strains were also looked up for the expression of efflux pump adeB and ade $J$ genes by quantitative real time PCR. The assay showed higher expression of adeB and adeJ genes (1.1-5.6 and 1.0-2.6 fold respectively) in $66 \%(n=74)$ and $42.8 \%(n=48)$ CRAB strains respectively, compared to A.baumannii ATCC 19606 strain (Figure 2).

\section{Quantitative real time experiment for the expression of car0, omp33-36, oprD porin genes}

When CRAB strains were analyzed for the expression of porin genes, 84 (75\%), $74(66 \%)$ and 81 (72.3\%) CRAB strains showed decreased carO, omp33-36 and oprD expression level respectively [expression levels being 0.06-0.90 fold, 0.01-0.93 fold and 0.01-0.97 fold, respectively] than that observed in A.baumannii ATCC 19606 strain (Figure 2).

\section{Correlation between altered expression of efflux pumps and porin genes with oxacillinase and metallo- $\beta$ - lactamases}

All isolates with increased expression of $\operatorname{ade} B(n=72)$ and adeJ $(n=48)$ were resistant to carbapenems. Among the isolates with increased of $a d e B$ expression, $87.8 \%(n=65)$ encoded bla $a_{\text {OXA-23-like, }} 44.6 \%(n=33)$ bla $a_{\text {OXA-24/40-like, }} 1.3 \%(\mathrm{n}=1)$ bla $a_{\mathrm{IMP}}$ and $8.1 \%(\mathrm{n}=6)$ bla $\mathrm{N}_{\mathrm{NDM}}$. Similar result was observed among isolates with adeJ over-expression, with $85.4 \%(n=41)$ encoding b/a $a_{\text {OXA-23-like, }} 43.7 \%(n=21) b / a_{\text {OXA-24/40-like, }} 0 \%$ $(\mathrm{n}=0) b / a_{\mathrm{IMP}}$ and $10.4 \%(\mathrm{n}=5)$ bla $\mathrm{NDM}_{\mathrm{N}}$, respectively (Figure 3). However, no significant correlation was observed between over expression ( $>1$ fold) of adeB and adeJ genes and resistance to carbapenems, when using either $\chi^{2}$ test or student's $t$ test.

All isolates with decreased expression of $\operatorname{carO}(\mathrm{n}=84)$, omp33-36 $(\mathrm{n}=74)$ and $\operatorname{oprD}(\mathrm{n}=81)$ were resistant to carbapenems. Among isolates with decreased of carO expression, $82.1 \%(n=69)$ carried bla $a_{\text {OXA-23- }}$ like, $31 \%(n=26)$ bla $/ a_{\text {XX-24/40-like, }} 5.9 \%(n=5) b / a_{\text {IMP }}$ and $5.9 \%(n=5) b / a_{\text {NDM }}$ genes. Alike outcome was observed among isolates with reduced omp33-36 expression, with $79.8 \%(n=59)$ encoding bla OXA-23-

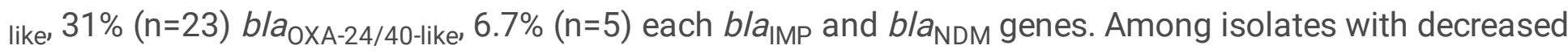
expression of $\operatorname{oprD} 81.4 \%(\mathrm{n}=66)$ carried bla OXA-23-like, $28.3 \%(\mathrm{n}=23)$ bla $\mathrm{OXA}_{2} 24 / 40$-like, $6.1 \%(\mathrm{n}=5)$ each $b / a_{\text {IMP }}$ and $b / a_{\mathrm{NDM}}$ genes (Figure 4). Compatible to outcomes of efflux pump genes, no significant correlation was observed between decreased expression ( $<1$ fold) of carO, omp33-36, oprD genes and resistance to carbapenems when using either $\chi^{2}$ test or Student's $t$ test.

\section{Sequence typing of CRAB strains}

CRAB strains were typed for knowing the epidemiological type of origin. Multiplex PCR performed for the identification of sequence groups (SGs) revealed 57 (50.9\%) CRAB strains belonged to (sequence group 1) SG1 [European clone (EU) clone II], majority $(85.9 \% ; n=49)$ being recovered from ICU patients. Seven $(6.3 \%)$ isolates belonged to SG2 (EU clone I) while, six (5.4\%) isolates belonged to the SG3 (EU clone III). Furthermore, 42 (37.5\%) isolates belonged to new variants of SGs (SG4-SG-9). No significant correlation was observed between carbapenemase genes distribution and the three main sequence groups (Table 1). 


\section{Discussion}

The present investigation was performed to search the plausible mechanisms implicated in the carbapenem resistance in A.baumannii. All A.baumannii obtained from diverse clinical infections were resistant to imipenem and meropenem leaving very few antibiotics as therapeutic modality. All these strains were MDR and half of them were XDR. Presence of bla OXA-23-like gene was as an exclusive basis of carbapenem resistance in A.baumannii strains. To the best of our knowledge, this is the first report of predominance of multiple CRAB strains harboring both OXA-23 and OXA-40 carbapenemase simultaneously from Iran. Over-expression of $a d e B$ and ade $J$ and decreased expression of carO, omp3336 and $o p r D$ was a marked feature in A.baumannii strains. Concurrent presence of OXA and MBLs genes with altered expression of efflux pump and porin genes was noticed in the CRAB strains.

In the present study, $86.6 \%$ A.baumannii isolates were obtained from patients admitted to ICU and only $13.4 \%$ were isolated from other wards. Research studies conducted previously in Iran (34-36) and other countries (37-39) have reported this prevalence to range from $28-74 \%$. The high prevalence in our study is probably due to the inclusion of five ICU wards.

All $A$. baumannii isolates were resistant to carbapenems, cephalosporins, fluoroquinolones and cotrimoxazole. This rate of antibiotic resistance is higher than similar studies performed in Iran whereby $60-100 \%$ resistance was reported $(34-36,40)$. While, resistance rates towards imipenem and/or meropenem in $A$. baumannii isolates from other countries vary from $42-85 \%(37,38,41,42)$, the highlevel resistance to carbapenem ( $\mathrm{MIC}>32 \mu \mathrm{g} / \mathrm{mL}$ ) observed in our study is compatible to studies conducted earlier in Iran, which show overuse of carbapenem has led to the emergence of CRAB strains $(34-36,40)$. This observation, of high rate carbapenem resistance is of considerable concern, because until recently this class of antibiotic was considered the drug of choice for the treatment of serious nosocomial infections caused by A.baumannii (1-3).

In this study, despite the resistance towards almost all antibiotics, colistin was effective against our A.baumannii isolates, a finding consistent with other Iranian studies $(34,36,40)$. This may be due to sparse usage of colistin, because of its side effects. However, following the emergence of resistance to carbapenem, colistin is one of the last options for the treatment of CRAB infections $(2,4)$. However, colistin usage has created emergence of resistance toward this antibiotic too $(37,38,41)$.

Carbapenem resistance in A.baumannii is most often mediated by acquired CHDLs (b/aXA-23-like, bla $a_{\text {OXA-24/40-like, }}$ b/a $a_{\text {OXA-58-like }}$ ) and less frequently by MBLs carbapenemase (5). The most disseminated CHDLs in the world is bla $a_{\mathrm{OXA}-23 \text {-like }}$ and A.baumannii clinical isolates producing this enzyme are frequently associated with hospital outbreaks $(5,6)$. In this study, bla OXA-23-like gene was the most common (82.1\%) OXA gene detected among CRAB strains, in agreement with similar studies from both Iran $(34,36,40)$ and other countries $(38,39,41,42)$. In the current study, the rate of bla OXA-24/40-like (36.6\%), was comparatively lower than $b / a_{\text {OXA-23-like. }}$ Though the prevalence of $b / a_{\text {OXA-24/40-like }}$ varies in 
Iranian studies from $1.6-68.5 \%(34,36,40)$, the prevalence is in moderate range $(5.4-57.6 \%)$ in other countries $(38,42,43)$.

The role of MBLs in resistance to carbapenems in A.baumannii is lower than OXAs and our results are consistent with that of previous studies from Turkey, Egypt and Spain $(39,42,43)$. Generally, the prevalence of metallo- $\beta$-lactamase in CRAB strains in comparison with oxacillinase is rather low and have been reported to vary from $0-29 \%(35-37,41,43)$. These variations may be related to different ecological situations, antibiotic therapy programs and antibiotic consumption patterns in different countries. Coexistence of three different bla OXA-like genes (b/aXX-23-like $/$ bla $/ a_{\text {OXA-51-like }} / b / a_{\text {OXA-24/40-like }}$ ) was observed in $46.3 \%$ A.baumannii strains (40).

Few studies have examined the impact of drug resistance as mediated by efflux pumps and porin in A.baumannii $(12,15,19,25,26,44)$. Efflux overexpression and porin down regulation are thought to be the major mechanisms of drug resistance in A.baumannii $(12,15)$. Expression of RND efflux pumps (AdeABC, AdelJK and AdeFGH) are known to be one of the major mechanisms of drug resistance in A.baumannii clinical isolates with a wide range of antibiotic substrate profile. These findings suggest that, in addition to enzymatic mechanisms of drug resistance, drug efflux may also have an impact in antibiotic resistance of $A$.baumannii isolates $(25,45,46)$. The expression levels of the porins CarO (47), OprD (13) and the OMP33-36 kDa (14) are also reportedly associated with antibiotic resistance. While no statistically significant correlations could be made between RND pump and porin expression with carbapenem resistance, presence of oxacillinase gene (bla OXA-23-like $)$ may be the main cause of resistance to carbapenem or interplay of efflux pumps and porin may have complementary roles in promoting resistance to carbapenems in these strains.

Research studies correlate relationship between down-regulation of carO porin expression and MDR (14, $15,48)$ however, no statistically significance was observed. The significance of $33-36 \mathrm{kDa}$ OMP has been suggested as an important virulence factor rather than an influential factor in carbapenem resistance (15). Similarly, OprD is part of a $P$. aeruginosa protein subgroup with homology to OprQ, involved in magnesium tolerance and functions in low iron environments but has no role in carbapenem resistance (16). Moreover, research from Brazil has shown that bla OXA-23-like $_{\text {is }}$ the main mechanism of carbapenem resistance and and the loss of porin CarO plays a minor role in this phenotype. Study from Argentina provided a supportive role of loss of CarO protein in carbapenem-resistant A.baumannii clinical isolates, recommending that CarO participates in the influx of these antibiotics. Del Mar Tomas et al. (14) showed that A.baumannii harboring the 33-36 kDa OMP led to a clear reduction in the MICs of imipenem and meropenem. Catel-Ferreira et al. (16) showed that the OprD homologue is not involved in specific antibiotic diffusion and instead functions similar to the Pseudomonas OprQ protein, offering specific binding sites for iron and magnesium ions and allowing A.baumannii to adapt to stress conditions.

\section{Conclusion}


Despite the diverse antibiotic susceptibility profile, carbapenems continue to play an important role in the treatment of severe illnesses caused by A.baumannii and clinicians continue to prescribe them if resistance does not constraint them. The overall results of this work are consistent with the multifactorial nature of carbapenem resistance in A.baumannii. The high distribution of class $D$ carbapenemase encoding genes, mainly b/aXX-23-like, is likely the main reason of carbapenem resistance in our hospital setting. Predominance of multiple CRAB strains harboring OXA-51, OXA-23 and OXA-40 carbapenemase simultaneously cannot be overlooked as these strains may be an alarming concern. Similarly, overexpression of $a d e B$ and $a d e J$ efflux pump genes and decreased expression of $c a r O$, omp33-36 and oprD genes simultaneously with the presence of OXA and/or MBL genes in an endemic hospital setting may be a distress in view of the dissemination of these strains.

\section{Abbreviations}

VAP: Ventilator Associated Pneumonia; ICUs: Intensive Care Units; WHO: World Health Organization; PBPs: Penicillin-Binding Proteins; MDR: Multi Drug Resistant; XDR: Extensive Drug Resistant; CRAB: Carbapenem Resistant A. baumannii ;MBLs: Metallo $\beta$-Lactamases; CHDLs: Carbapenem Hydrolyzing class D $\beta$-Lactamases; IS: Insersion Sequence; SGs: Sequence Groups; EC: European Clone; CLSI: Clinical and Laboratory Standards Institute; MIC: Minimum Inhibitory Concentration; RND: Resistance Nodulation Cell Division; AdeABC: Acinetobacterdrug efflux; ATCC: American Type Culture Collection; TBE: Tris Borate EDTA; bp: base pair

\section{Declarations}

\section{Ethics approval and consent to participate}

Ethical Committee of Tabriz University of Medical Sciences approved the project under the code: IR.TBZ MED.VCR.REC.1397.042 dated 1396/11/04

\section{Consent for publication}

Not Applicable

\section{Availability of data and material}

Not Applicable

\section{Competing interests}

The authors declare no conflict of interest

\section{Funding}


This work was supported by Immunology Research Center, Faculty of Medicine, Tabriz University of Medical Sciences, Tabriz, I. R. Iran

\section{Authors' contributions}

Abolfazl Vahhabi and Alka Hasani conceived and planned the experiments. Abolfazl Vahhabi and Faeze Abbaszadeh carried out the experiments. Alka Hasani, Mohammad Ahangarzadeh Rezaee and Hossein Samadi Kafil contributed to sample preparation. Abolfazl Vahhabi, Behzad Baradaran and Akbar Hasani contributed to the interpretation of the results. Abolfazl Vahhabi and Alka Hasani took the lead in writing the manuscript. Alka Hasani supervised the project. All authors provided critical feedback and helped shape the research, analysis and manuscript.

\section{Acknowledgments}

We are indebted to Dr. Faye Morris for critical reading of the manuscript and insightful suggestions keeping with important intellectual content. We appreciate the meticulous attention of Ms. Leila Dehghani and Mr. Vahid Sharifzadeh Peyvasti in the collection of clinical isolates and phenotypic workup. This work was supported by Immunology Research Center, Faculty of Medicine, Tabriz University of Medical Sciences, Tabriz, I. R. Iran.

This is a report of a database from $\mathrm{PhD}$ thesis of the corresponding author registered in the Tabriz University of Medical Sciences (Thesis No-59781).

\section{References}

1. Peleg AY, Seifert H, Paterson DL. Acinetobacter baumannii: emergence of a successful pathogen. Clin Microbiol Rev. 2008;21(3):538-82.

2. Giamarellou H, Antoniadou A, Kanellakopoulou K. Acinetobacter baumannir: a universal threat to public health? Int J Antimicrob Agents. 2008;32(2):106-19.

3. Eliopoulos GM, Maragakis LL, Perl TM. Acinetobacter baumannir: epidemiology, antimicrobial resistance, and treatment options. Clin Infect Dis. 2008;46(8):1254-63.

4. Gordon NC, Wareham DW. Multidrug-resistant Acinetobacter baumannii: mechanisms of virulence and resistance. Int J Antimicrob Agents. 2010;35(3):219-26.

5. Poirel L, Nordmann P. Carbapenem resistance in Acinetobacter baumannir: mechanisms and epidemiology. Clin Microbiol Infect. 2006;12(9):826-36.

6. Poirel L, Naas T, Nordmann P. Diversity, epidemiology, and genetics of class D $\beta$-lactamases. Antimicrob Agents Chemother. 2010;54(1):24-38.

7. Lin M-F, Lin Y-Y, Tu C-C, Lan C-Y. Distribution of different efflux pump genes in clinical isolates of multidrug-resistant Acinetobacter baumannii and their correlation with antimicrobial resistance. $J$ Microbiol Immunol Infect. 2017;50(2):224-31. 
8. Vila J, Martí S, Sanchez-Céspedes J. Porins, efflux pumps and multidrug resistance in Acinetobacter baumannii. J Antimicrob Chemother. 2007;59(6):1210-5.

9. Yoon E-J, Chabane YN, Goussard S, Snesrud E, Courvalin P, Dé E, et al. Contribution of resistancenodulation-cell division efflux systems to antibiotic resistance and biofilm formation in Acinetobacter baumannii. MBio. 2015;6(2).

10. Marchand I, Damier-Piolle L, Courvalin P, Lambert T. Expression of the RND-type efflux pump AdeABC in Acinetobacter baumannii is regulated by the AdeRS two-component system. Antimicrob Agents Chemother. 2004;48(9):3298-304.

11. Wieczorek P, Sacha P, Hauschild T, Zórawski M, Krawczyk M, Tryniszewska E. Multidrug resistant Acinetobacter baumannii-the role of AdeABC (RND family) efflux pump in resistance to antibiotics. Folia Histochem Cytobiol. 2008;46(3):257-67.

12. Fernández L, Hancock RE. Adaptive and mutational resistance: role of porins and efflux pumps in drug resistance. Clin Microbiol Rev. 2012;25(4):661-81.

13. Siroy A, Molle V, Lemaître-Guillier C, Vallenet D, Pestel-Caron M, Cozzone AJ, et al. Channel formation by CarO, the carbapenem resistance-associated outer membrane protein of Acinetobacter baumannii. Antimicrob Agents Chemother. 2005;49(12):4876-83.

14. Del Mar Tomás M, Beceiro A, Pérez A, Velasco D, Moure R, Villanueva R, et al. Cloning and functional analysis of the gene encoding the 33-to 36-kilodalton outer membrane protein associated with carbapenem resistance in Acinetobacter baumannii. Antimicrob Agents Chemother. 2005;49(12):5172-5.

15. Rumbo C, Gato E, López M, De Alegria CR, Fernández-Cuenca F, Martínez-Martínez L, et al. Contribution of efflux pumps, porins, and $\beta$-lactamases to multidrug resistance in clinical isolates of Acinetobacter baumannii. Antimicrob Agents Chemother. 2013;57(11):5247-57.

16. Catel-Ferreira M, Nehmé R, Molle V, Aranda J, Bouffartigues E, Chevalier S, et al. Deciphering the function of the outer membrane protein OprD homologue of Acinetobacter baumannii. Antimicrob Agents Chemother. 2012;56(7):3826-32.

17. Fernández-Cuenca F, Smani Y, Gómez-Sánchez MC, Docobo-Pérez F, Caballero-Moyano FJ, Domínguez-Herrera J, et al. Attenuated virulence of a slow-growing pandrug-resistant Acinetobacter baumannii is associated with decreased expression of genes encoding the porins CarO and OprDlike. Int J Antimicrob Agents. 2011;38(6):548-9.

18. Dupont $M$, Pagès J-M, Lafitte $D$, Siroy A, Bollet $C$. Identification of an OprD Homologue in Acinetobacter baumannii. J Proteome Res. 2005;4(6):2386-90.

19. Bratu S, Landman D, Martin DA, Georgescu C, Quale J. Correlation of antimicrobial resistance with $\beta$ lactamases, the OmpA-like porin, and efflux pumps in clinical isolates of Acinetobacter baumannii endemic to New York City. Antimicrob Agents Chemother. 2008;52(9):2999-3005.

20. Hall GS. Bailey \& Scott's Diagnostic Microbiology, 13th Edn: American Society for Clinical Pathology; 2013. 
21. Higgins PG, Lehmann M, Wisplinghoff H, Seifert H. gyrB multiplex PCR to differentiate between Acinetobacter calcoaceticus and Acinetobacter genomic species 3. J Clin Microbiol. 2010;48(12):4592-4.

22. Gundi VA, Dijkshoorn L, Burignat S, Raoult D, La Scola B. Validation of partial rpoB gene sequence analysis for the identification of clinically important and emerging Acinetobacterspecies. Microbiology. 2009;155(7):2333-41.

23. Clinical and Laboratory Standards Institute. Performance standards for antimicrobial susceptibility testing. 28th ed. CLSI supplement M100s. CLSI; 2018.

24. Magiorakos AP, Srinivasan A, Carey R, Carmeli Y, Falagas M, Giske C, et al. Multidrug-resistant, extensively drug-resistant and pandrug-resistant bacteria: an international expert proposal for interim standard definitions for acquired resistance. Clin Microbiol Infect. 2012;18(3):268-81.

25. Lin L, Ling B-D, Li X-Z. Distribution of the multidrug efflux pump genes, adeABC, adeDE and adelJK, and class 1 integron genes in multiple-antimicrobial-resistant clinical isolates of Acinetobacter baumannii-Acinetobacter calcoaceticus complex. Int J Antimicrob Agents. 2009;33(1):27-32.

26. Zhang Y, Li Z, He X, Ding F, Wu W, Luo Y, et al. Overproduction of efflux pumps caused reduced susceptibility to carbapenem under consecutive imipenem-selected stress in Acinetobacter baumannii. Infect Drug Resist. 2017;11:457.

27. Woodford N, Ellington MJ, Coelho JM, Turton JF, Ward ME, Brown S, et al. Multiplex PCR for genes encoding prevalent OXA carbapenemases in Acinetobacterspp. Int J Antimicrob Agents. 2006;27(4):351-3.

28. Higgins PG, Poirel L, Lehmann M, Nordmann P, Seifert H. OXA-143, a novel carbapenem-hydrolyzing class D $\beta$-lactamase in Acinetobacter baumannii. Antimicrob Agents Chemother. 2009;53(12):50358.

29. Higgins PG, Pérez-Llarena FJ, Zander E, Fernández A, Bou G, Seifert H. OXA-235, a novel class D $\beta$ lactamase involved in resistance to carbapenems in Acinetobacter baumannii. Antimicrob Agents Chemother. 2013;57(5):2121-6.

30. Turton JF, Ward ME, Woodford N, Kaufmann ME, Pike R, Livermore DM, et al. The role of ISAba 1 in expression of OXA carbapenemase genes in Acinetobacter baumannii. FEMS Microbiol Lett. 2006;258(1):72-7.

31. Poirel L, Walsh TR, Cuvillier V, Nordmann P. Multiplex PCR for detection of acquired carbapenemase genes. Diagn Microbiol Infect Dis. 2011;70(1):119-23.

32. Nordmann P, Poirel L, Carrër A, Toleman MA, Walsh TR. How to detect NDM-1 producers. J Clin Microbiol. 2011;49(2):718-21.

33. Turton J, Gabriel S, Valderrey C, Kaufmann M, Pitt T. Use of sequence-based typing and multiplex PCR to identify clonal lineages of outbreak strains of Acinetobacter baumannii. Clin Microbiol Infect. 2007;13(8):807-15.

34. Sohrabi N, Farajnia S, Akhi MT, Nahaei MR, Naghili B, Peymani A, et al. Prevalence of OXA-type $\beta$ lactamases among Acinetobacter baumannii isolates from Northwest of Iran. Microb Drug Resist. 
2012;18(4):385-9.

35. Maspi H, Hosseini HM, Amin M, Fooladi AAl. High prevalence of extensively drug-resistant and metallo $\beta$-lactamase-producing clinical Acinetobacter baumannii in Iran. Microb Pathog. 2016;98:155-9.

36. Shoja S, Moosavian M, Rostami S, Abbasi F, Tabatabaiefar MA, Peymani A. Characterization of oxacillinase and metallo- $\beta$-lactamas genes and molecular typing of clinical isolates of Acinetobacter baumannii in Ahvaz, South-West of Iran. Jundishapur J Microbiol. 2016;9(5).

37. Rynga D, Shariff M, Deb M. Phenotypic and molecular characterization of clinical isolates of Acinetobacter baumannii isolated from Delhi, India. Ann Clin Microbiol Antimicrob. 2015;14(1):40.

38. Elabd FM, Al-Ayed MS, Asaad AM, Alsareii SA, Qureshi MA, Musa HA-A. Molecular characterization of oxacillinases among carbapenem-resistant Acinetobacter baumannii nosocomial isolates in a Saudi hospital. J Infect Public Health. 2015;8(3):242-7.

39. Aksoy MD, Çavuşlu Ş, Tuğrul HM. Investigation of metallo $\beta$-lactamases and oxacilinases in carbapenem resistant Acinetobacter baumannii strains isolated from inpatients. Balkan Med J. 2015;32(1):79-83.

40. Sarhaddi N, Soleimanpour S, Farsiani H, Mosavat A, Dolatabadi S, Salimizand H, et al. Elevated prevalence of multidrug-resistant Acinetobacter baumannii with extensive genetic diversity in the largest burn centre of northeast Iran. J Glob Antimicrob Resist. 2017;8:60-6.

41. Al-Sweih N, Al-Hubail M, Rotimi V. Three distinct clones of carbapenem-resistant Acinetobacter baumannii with high diversity of carbapenemases isolated from patients in two hospitals in Kuwait. J Infect Public Health. 2012;5(1):102-8.

42. Khorsi K, Messai Y, Hamidi M, Ammari H, Bakour R. High prevalence of multidrug-resistance in Acinetobacter baumannii and dissemination of carbapenemase-encoding genes bla OXA-23-like, $_{\text {, }}$ /aXA24-like and bla NDM-1 $_{1}$ in Algiers hospitals. Asian Pac J Trop Med. 2015;8(6):438-46.

43. Villalón P, Valdezate S, Medina-Pascual MJ, Carrasco G, Vindel A, Saez-Nieto JA. Epidemiology of the Acinetobacter-derived cephalosporinase, carbapenem-hydrolysing oxacillinase and metallo- $\beta$ lactamase genes, and of common insertion sequences, in epidemic clones of Acinetobacter baumannii from Spain. J Antimicrob Chemother. 2012;68(3):550-3.

44. Zhu L-J, Pan Y, Gao C-Y, Hou P-F. Distribution of Carbapenemases and Efflux Pump in Carbapenemresistance Acinetobacter baumannii. Ann Clin Lab Sci. 2020;50(2):241-6.

45. Damier-Piolle L, Magnet S, Brémont S, Lambert T, Courvalin P. AdelJK, a resistance-nodulation-cell division pump effluxing multiple antibiotics in Acinetobacter baumannii. Antimicrob Agents Chemother. 2008;52(2):557-62.

46. Gaddy JA, Tomaras AP, Actis LA. The Acinetobacter baumannii 19606 OmpA protein plays a role in biofilm formation on abiotic surfaces and in the interaction of this pathogen with eukaryotic cells. Infect Immun. 2009;77(8):3150-60.

47. Mussi MA, Limansky AS, Viale AM. Acquisition of resistance to carbapenems in multidrug-resistant clinical strains of Acinetobacter baumannii: natural insertional inactivation of a gene encoding a 
member of a novel family of $\beta$-barrel outer membrane proteins. Antimicrob Agents Chemother. 2005;49(4):1432-40.

48. Limansky AS, Mussi MA, Viale AM. Loss of a 29-kilodalton outer membrane protein in Acinetobacter baumannii is associated with imipenem resistance. J Clin Microbiol. 2002;40(12):4776-8.

\section{Tables}

Table 1. Distribution of carbapenemase genes in various sequence groups 


\begin{tabular}{|c|c|}
\hline Clonal group (European clone) $(\mathrm{N})$ & Carbapenemase gene (\%) \\
\hline $\begin{array}{l}\text { SG1 } \\
(E C \text { II) } \\
(57)\end{array}$ & 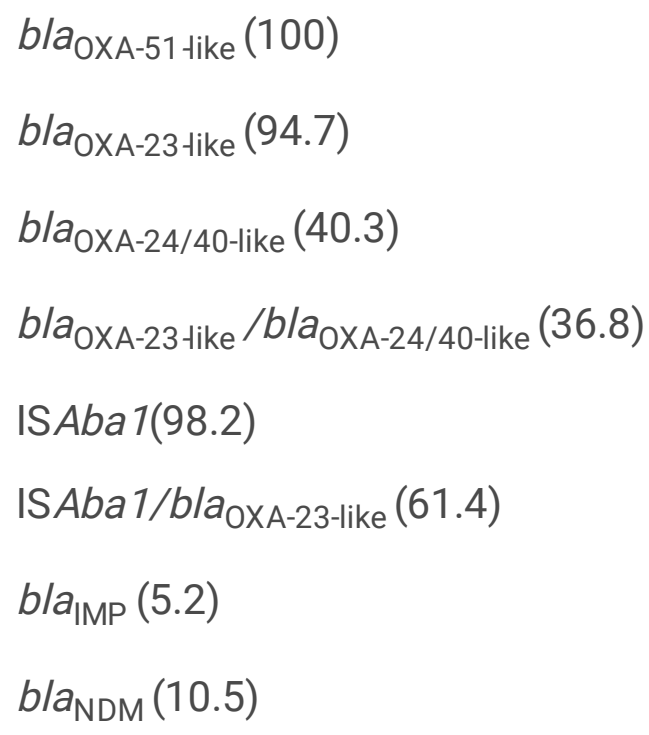 \\
\hline $\begin{array}{l}S G 2 \\
(E C I) \\
(7)\end{array}$ & 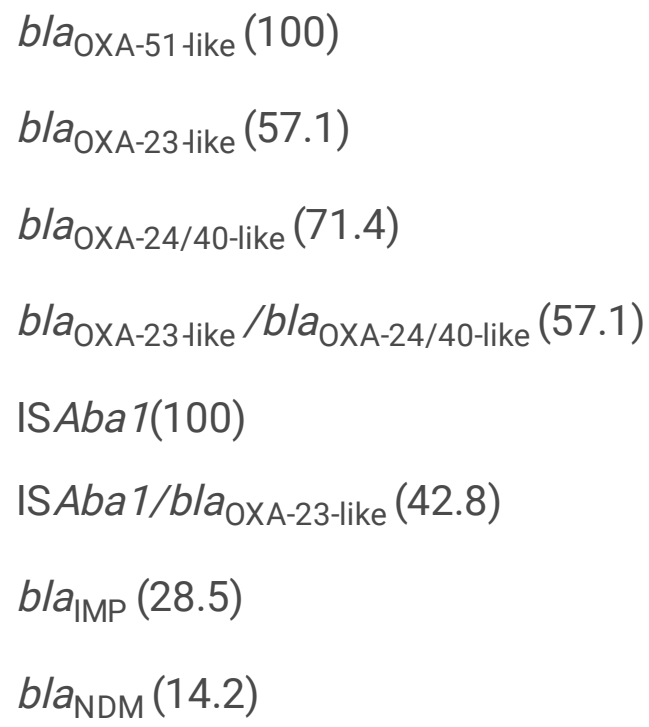 \\
\hline $\begin{array}{l}\text { SG3 } \\
\text { (EC III) } \\
(6)\end{array}$ & $\begin{array}{l}\text { bla OXA-51 tike }(100) \\
\text { bla OXA-23-like }(100) \\
\text { IS Aba 1(100) } \\
\text { ISAba 1/bla OXA-23-like }(100)\end{array}$ \\
\hline $\begin{array}{l}\text { SG4-SG9 } \\
\text { (Non SG 1, 2, 3) } \\
(42)\end{array}$ & 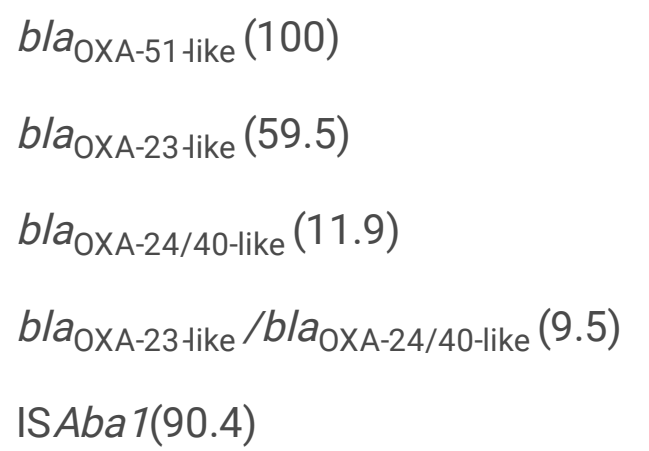 \\
\hline
\end{tabular}




\section{Figures}

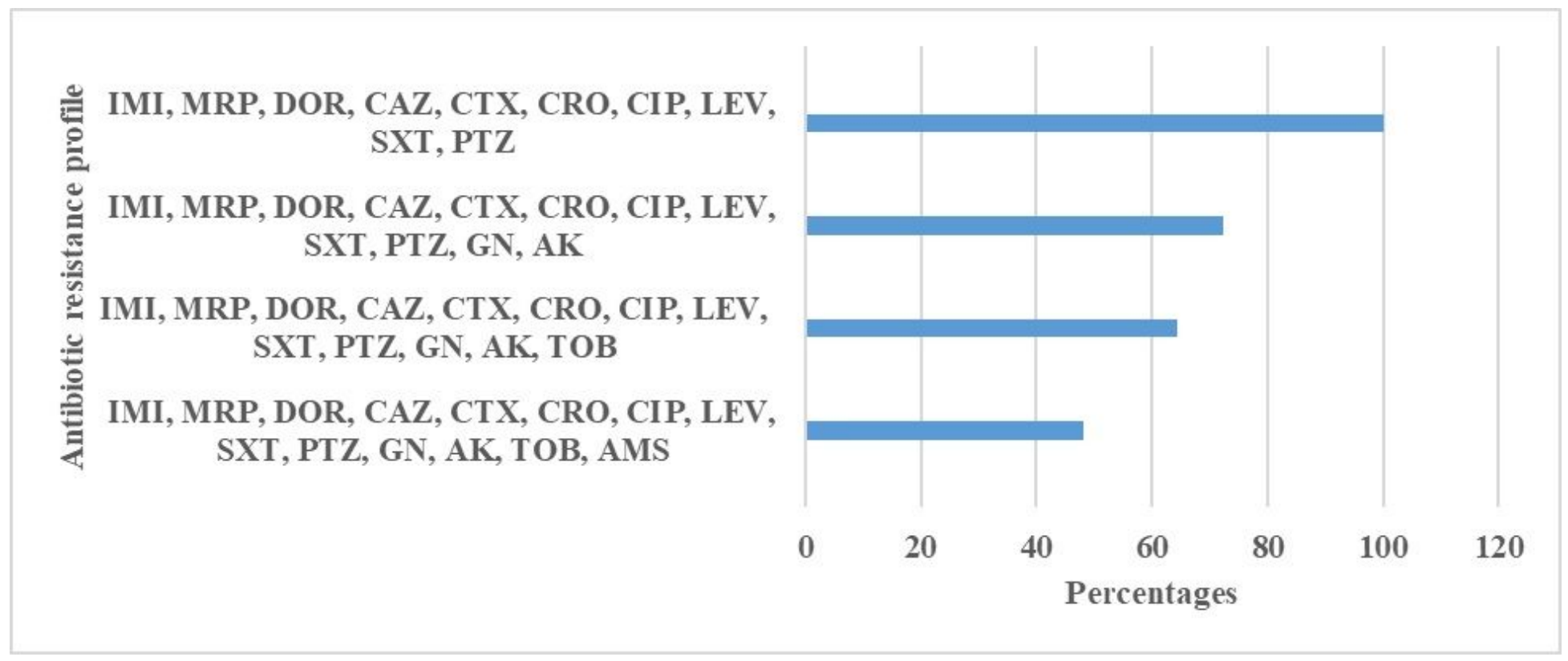

Figure 1

Antimicrobial resistance profile of A.baumannii IMI: Imipenem, MRP: Meropenem, DOR: Doripenem, CAZ: Ceftazidime, CTX: Cefotaxime, CRO: Ceftriaxone, CIP: Cip -rofloxacin, LEV: Levofloxacin, SXT: Cotrimoxazole, PTZ: piperacillin/tazobactam, GM: Gentamicin, AK: Amikacin, TOB: Tobramycin, AMS: Ampicillin/sulbactam 


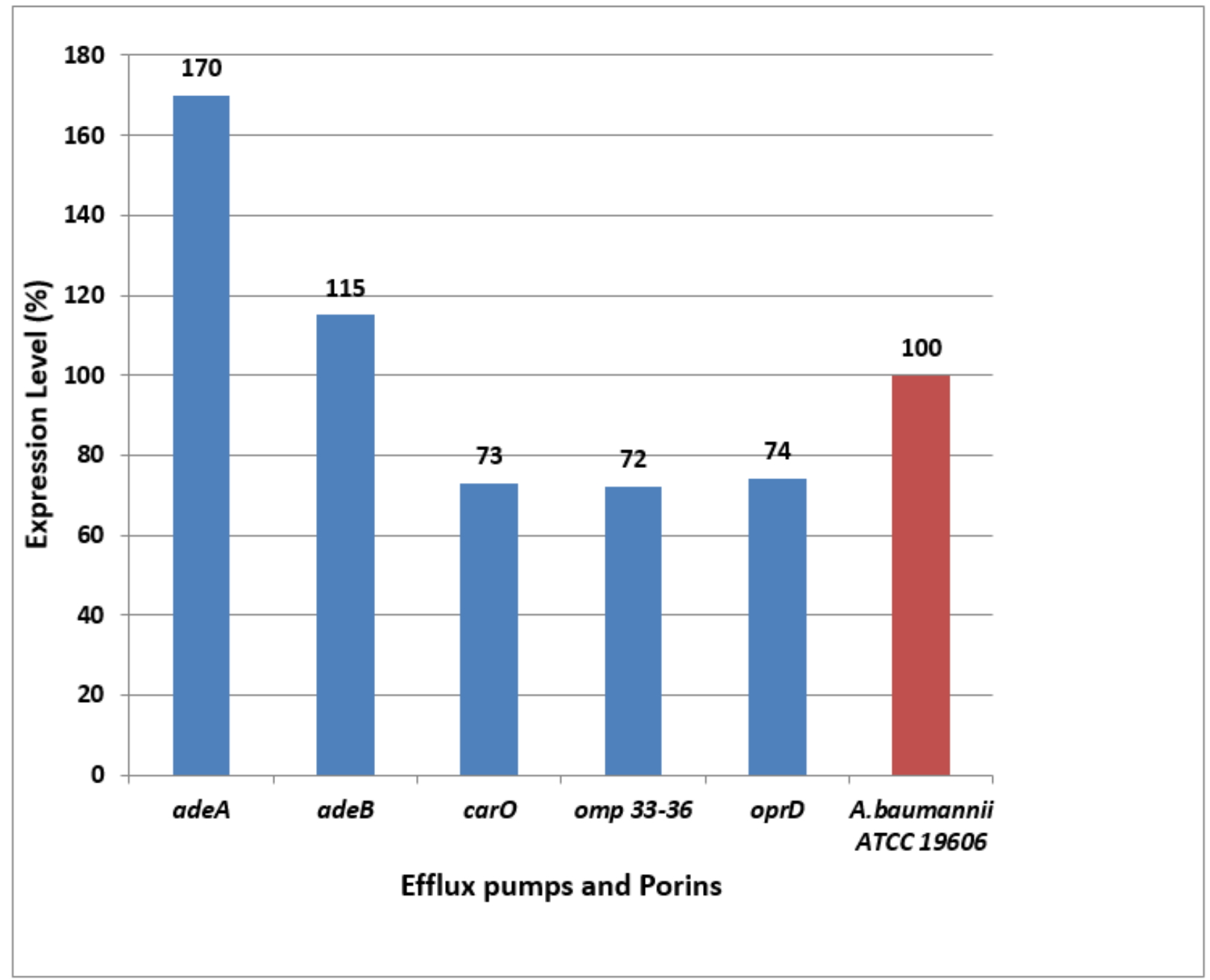

Figure 2

Relative expression of efflux pumps (adeB, adeJ) and porin (carO, omp 33-36, oprD) genes 


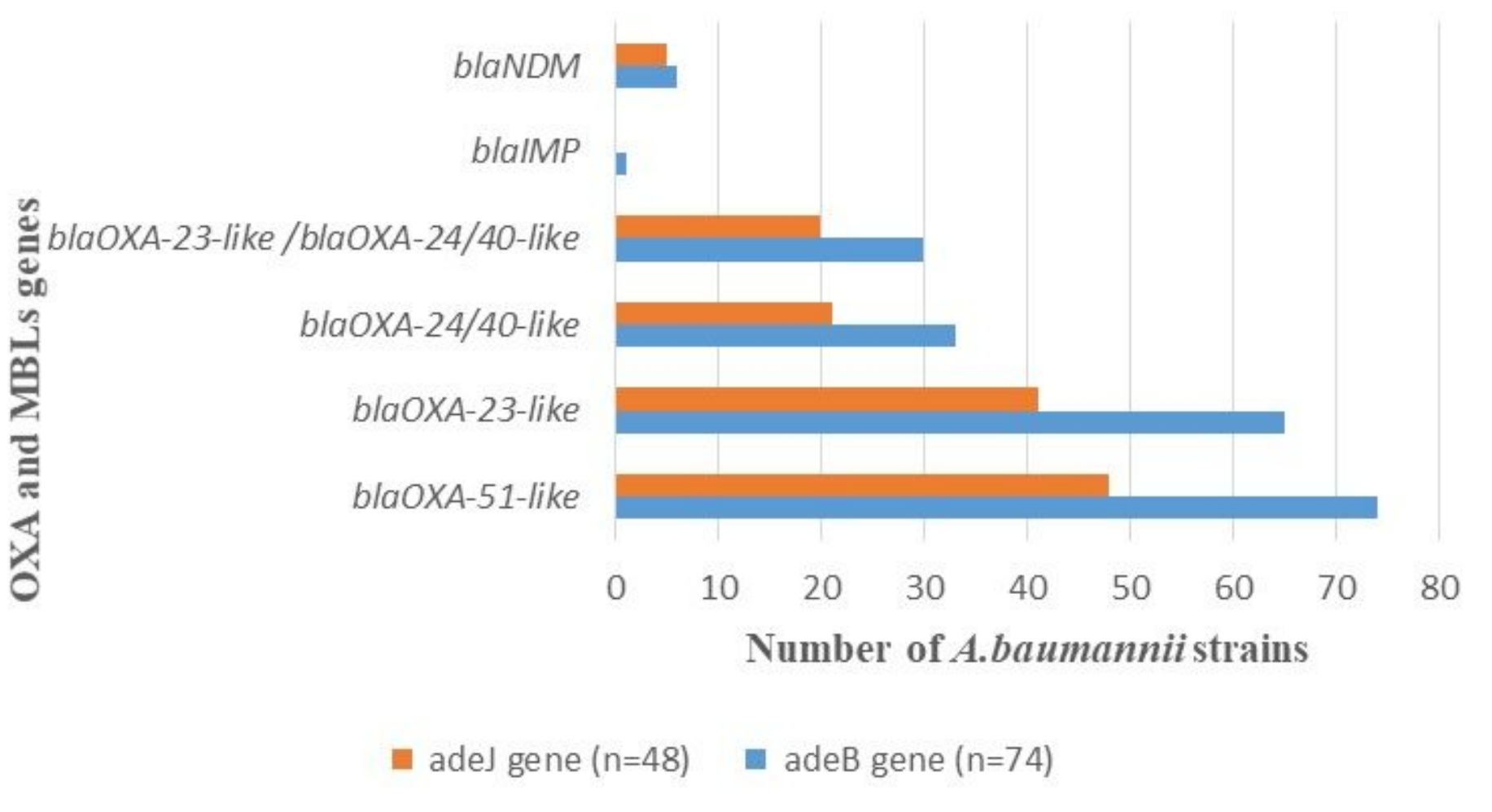

\section{Figure 3}

Correlation between increased expression of efflux pump genes and oxacillinase and metallo-beta lactamases

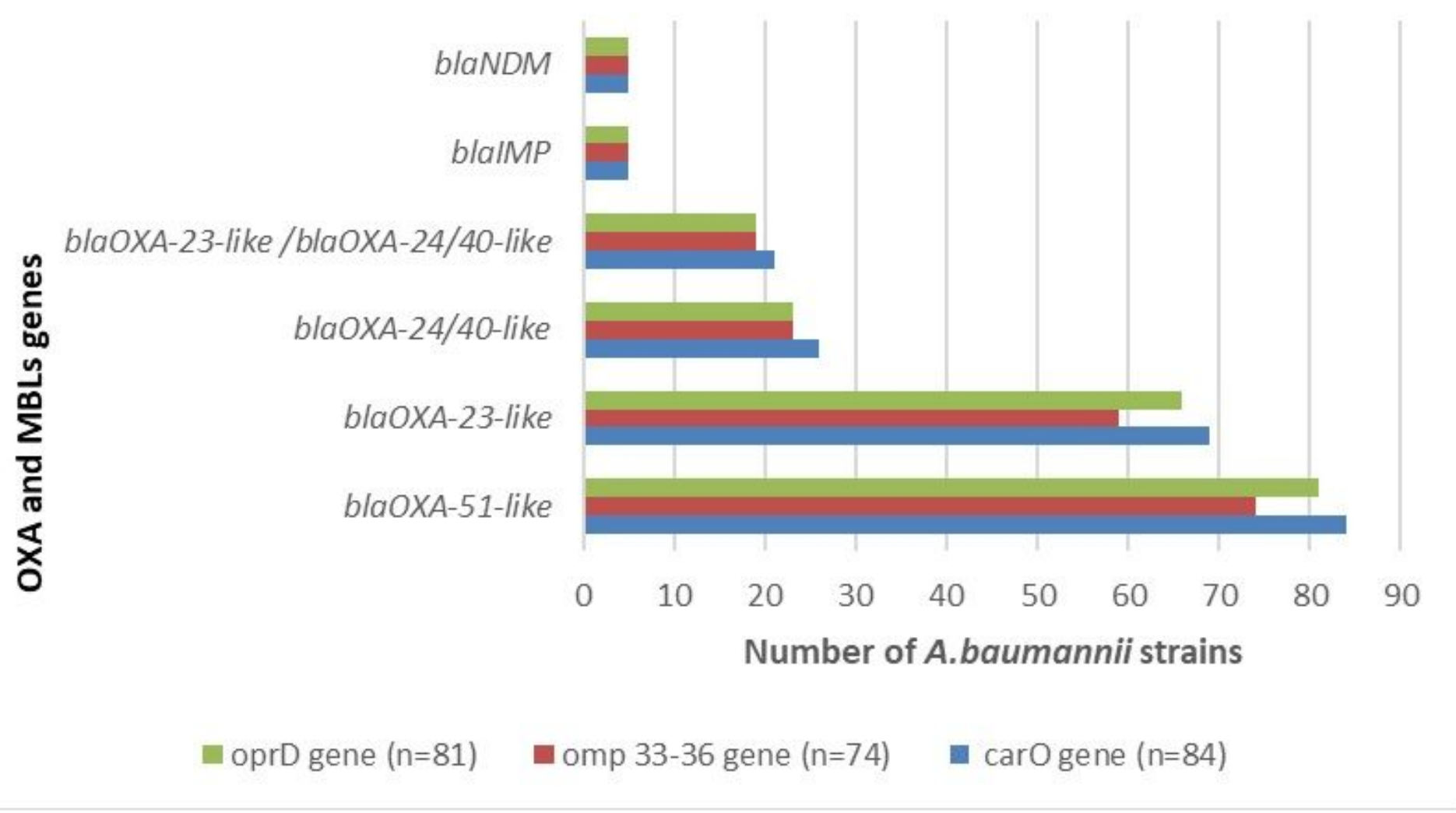


Correlation between decreased expression of porin genes and oxacillinase and metallo-beta lactamases

\section{Supplementary Files}

This is a list of supplementary files associated with this preprint. Click to download.

- SupplementryTables.doc 